\title{
The Effects of Thyroid Hormones and Their Abnormalities on Intestinal and Hepatic Glucose Metabolism
}

\author{
Dr. Ali Abdulateef Hasan Al-bayati ${ }^{1 *}$, Dr. Shatha MJ AL-Khateeb ${ }^{2}$ \\ ${ }^{1}(\mathrm{MD}, \mathrm{PhD}$ Newcastle), Department of Chemistry and Biochemistry, College of Medicine, Al- Mustansiriyah University, Baghdad, \\ Iraq \\ ${ }^{2}$ Assistant Professor, (PhD Clinical Biochemistry), Department of Chemistry and Biochemistry, College of Medicine, Al- \\ Mustansiriyah University, Baghdad, Iraq
}

DOI: $10.36348 / \mathrm{sijb} .2021 . v 04 \mathrm{i} 03.002$

| Received: 26.02.2021 | Accepted: 15.04.2021 | Published: 19.04.2021

*Corresponding author: Dr. Ali Abdulateef Hassan Al-bayati

Abstract

Thyroid diseases are a common endocrine disorder, and thyroid hormones physiologically are well-known to have direct and indirect effects on metabolic abnormalities. Disorders of carbohydrates (glucose) metabolism are frequently reported in both hypothyroidism and hyperthyroidism. This review considered to examine and analyze the role of thyroid hormones and their abnormalities in different level of glucose metabolism in intestinal and liver cells. A broad literature search been performed on Google Scholar and PubMed for the periods between 01/01/2021 and 25/03/2021. The search includes the followings key words; thyroid hormones, glucose metabolism, hepatocytes, hypothyroidism, hyperthyroidism, thyroid receptors. Liver represents a major organ that involves in both consumption, storage and production of glucose. The expression of thyroid hormones receptors in the hepatocytes are often recoded. Thyroid hormones facilitate glucose absorption from gastrointestinal system. In hyperthyroidism, hepatic glucose intake, glucokinase activity, mitochondrial activity and biogenesis, gluconeogenesis, glycogenolysis and hepatic glucose production all shown to increase. In hypothyroidism, intestinal Glucose Absorption, GLUT2 expression in hepatocytes, hepatic glucose intake, glucokinase activity, mitochondrial activity and biogenesis, gluconeogenesis, glycogenolysis are all seeming to reduced. In conclusion; Thyroid hormones represents a major regulator of glucose metabolism, and achieve their effects through promotion or demotion of expression or activation of enzymes responsible for regulation of different metabolic pathways. Thyroid disorders is associated with abnormal metabolism that could disturb the metabolic pathways and contributes or exacerbate other concomitant disease such as diabetes and metabolic syndrome.

Keywords: Thyroid hormones, glucose metabolism, hepatocytes, hypothyroidism, hyperthyroidism, thyroid receptors, thyroxin, triiodothyronine.

Copyright (C) 2021 The Author(s): This is an open-access article distributed under the terms of the Creative Commons Attribution 4.0 International License (CC BY-NC 4.0) which permits unrestricted use, distribution, and reproduction in any medium for non-commercial use provided the original author and source are credited.

\section{INTRODUCTION}

Thyroid hormones (THs) regarded as a major regulator of metabolic processes and energy expenditure of the body of animals and humans [1]. THs abnormalities is usually associated with body weight variations, fatigue and changes in heat generation that collectively represent indications of metabolic dysregulation [2]. Definitely, human cases of excess of THs "hyperthyroidism" are presented with increase in basal metabolic rate (BMR) and usually accompanied by weight loss while THs deficiency "hypothyroidism" characterized by decreased BMR and weight gain [3]. The role of THs on metabolic regulations comprises stimulation of different catabolic and anabolic processes of proteins, lipids and carbohydrates [1]. For example, in hyperthyroidism, stimulation of catabolic reactions is obvious represented by increased lipolysis and glycogenolysis [4], conversely, in hypothyroidism, inhibition of catabolic pathways is noticeable in decreased lipolysis and glycogenolysis [5]. Thus, the crucial energy controlling metabolic pathways are greatly affected by THs, and it is evident that any dysregulation of these hormones would markedly affect metabolism [6] and increasing the risk of getting diseases $[7,8]$.

\section{Effects of THs on glucose homeostasis}

Regulation of blood glucose level is a result of complex metabolic processes. These are starting with, nutrients intake, insulin secretion and action at a target tissue level and insulin counter regulatory hormones action. Disturbances of these factors can lead to 
metabolic imbalance featured as diabetes and metabolic syndrome [9].

Physiologically, T3 acts as a regulator of $\beta$ cell function through its effects on glucose uptake into pancreatic $\beta$ cells thereby controlling insulin secretion. Additionally, THs affect glucose metabolism in insulin responsive tissues (liver, adipose tissue and skeletal muscle) [10].

In the liver, THs antagonizing the effect of insulin causing hepatic insulin resistance by increasing glucose output. THs increase expression of glucose transporter (GLUT2) in hepatocytes and increase both glycogenolysis and gluconeogenesis [11]. Conversely, in peripheral tissues ((skeletal muscles and adipose tissues), THs shown to potentiates the effects of insulin by increasing cellular uptake of glucose and enhancement of glucose oxidation [10].

In hyperthyroidism, due to increased hepatic glucose production, glucose intolerance is prevalent [12]. Furthermore, excess of THs is associated with increased both fasting and postprandial insulin levels [13]. The $\beta$ cell apoptosis is markedly increased in cases of thyrotoxicosis that further deteriorates glucose tolerance [14]. Moreover, hyperthyroidism status can increase Peripheral insulin resistance via induction of production of proinflammatory mediators from adipose tissues such as TNF $\alpha$, adepokines and IL-6 [15].

In cases of reduced THs (hypothyroidism), variable glucose metabolic abnormalities been observed and contributed to further deteriorations of glucose intolerance. Absorption of glucose from intestine is impaired, glucose utilization by different tissues was reduced, muscle and liver glucose production was decreased, and normal or impaired liver glucose output are both reported [7].

Therefore, both hyper and hypothyroid status seem to impact glucose metabolism as shown in Table1 and tightly link to glucose intolerance and diabetes mellitus. In this review we will focus on effects of THs and their abnormalities on intestinal and hepatic glucose metabolism.

Table-1: Effects of thyroid status on different processes involving glucose metabolism. Variable response in hyperthyroidism and hypothyroidism in comparison to euthyroid state

\begin{tabular}{|l|l|l|}
\hline Processes & Hyperthyroidism & Hypothyroidism \\
\hline Intestinal Glucose Absorption & Increased & Decreased \\
\hline GLUT2 expression in hepatocytes & Increased & Decreased \\
\hline Hepatic glucose intake & Increased & Decreased \\
\hline Hepatic glucokinase activity & Enhanced & Reduced \\
\hline Glycogen synthesis & Decreased & Increased \\
\hline Glycogen content in hepatocyte & Reduced & Enhanced \\
\hline Enzymes activity of Pentose phosphate pathway & Enhanced & Reduced \\
\hline Glycolysis & No differences or reduced & No differences \\
\hline Pyruvate dehydrogenase enzyme expression and activity & Reduced & Reduced \\
\hline Mitochondrial activity & Highly increased & Decreased \\
\hline Mitochondrial biogenesis & Highly increased & Decreased \\
\hline Gluconeogenesis & Highly increased & Decreased \\
\hline Glycogenolysis & Highly increased & Decreased \\
\hline Hepatic glucose production & Highly increased & Decreased \\
\hline
\end{tabular}

\section{Synthesis, transport and control of THs}

THs are produced in thyroid gland. Thyroxine (T4) and Triiodothyronine (T3) are the two THs that secreted from follicular cells( the functional units of the thyroid) while calcitonin hormone secreted by parafollicular cells of the thyroid [16]. The synthesis of main THS (T3 and T4) by follicular cells required four essential components; tyrosine amino acids from follicular thyroglobulin, Iodine molecules from the diet, thyroperoxidase enzyme in the apical surface of follicular cells and hormonal stimulation represented by thyroid stimulating hormone (TSH) from anterior pituitary glands [17]. The formed iodotyrosine coupled to form either tetraiodothyronine, thyroxin, (T4) when the two diiodotyrosine assembled together or triiodothyronine (T3) when one diiodotyrosine binds to one monoiodotyrosine [17]. The T4 represents the major hormone secreted from the thyroid gland, but minority of hormone produced represents T3. However, $\mathrm{T} 3$ is the active form of THs at cellular level and the bulk of produced T3 in peripheral tissues, especially liver and kidney, by deiodination of $\mathrm{T} 4$ that achieved by type 2 (5-deiodinase) enzyme and this conversion represents the modulatory step of THs cellular availability [18]. The majority of secreted THs into blood streams are transported to different target tissues via binding to transport proteins (Thyroxine binding globulin (TBG), Thyroxine binding prealbumin (TBPA) and albumin). Also a small fraction of the THs present as free (unbound) and it is biologically active [19]. TSH from the anterior pituitary gland represents the major stimulus for THs production and release. Through 
binding to its receptor on the follicular cells, TSH promotes expression and synthesis of iodine transporter, thyroid peroxidase and thyroglobulin [20]. Classical regulation of the THs synthesis and release is under control of the hypothalamic-pituitary-thyroid axis (Fig1). When the circulatory level of THs is low, this will trigger a negative feedback that results in secretion of thyrotropin releasing hormone (TRH) from the hypothalamus and thyroid stimulating hormone (TSH) from the anterior pituitary gland [21]. Peripheral tissues also can regulate THs action via cell-autonomous regulation of plasma membrane THs transporters, deiodinase enzymes and cellular thyroid hormone receptors (THr) [22].

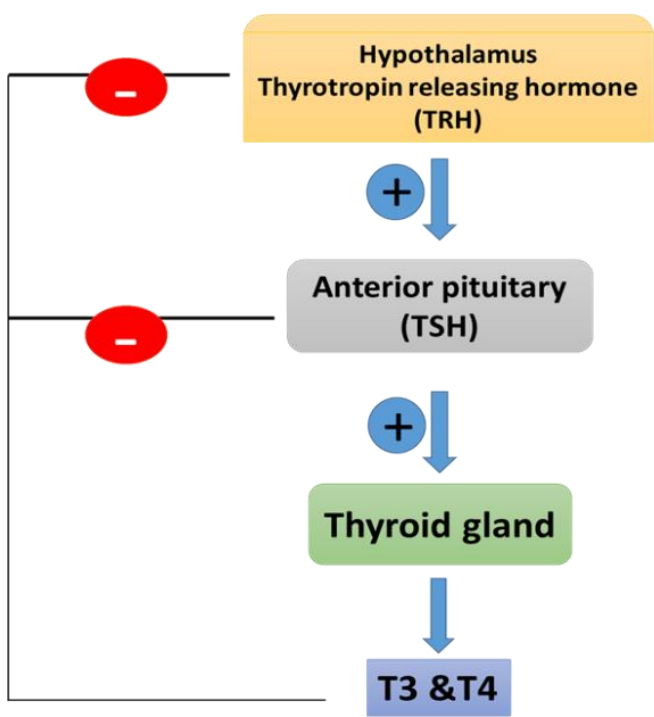

Fig-1: Schematic graph of the hypothalamic-pituitarythyroid axis. Hypothalamus release TRH in response to higher center orders, TRH has a direct stimulatory effects on anterior pituitary causing release of TSH. Through circulation, TSH reaching thyroid gland and play crucial role in THs production and release. T3 \&T4 are the main hormones released into the blood reaching peripheral tissues and their blood level triggers a negative feedback Activity on both hypothalamus and anterior pituitary to reduce their secretions of TRH and TSH

\section{Cellular THs transport, metabolism and action}

It was widely accepted for many years that, THs transport through plasma membrane of target tissue cells via simple diffusion depending on the lipophilic property (permit easy crossing of the plasma membrane lipid bilayer) of iodothyronines compounds. Recently, it is obvious that simple diffusion plays only a minor role, and specific carrier-mediated mechanisms had increasingly emerge as an acceptable way for THs transport across plasma membrane [16]. Up to date, in human, about sixteen transporters are identified to be involved in THs passages across plasma membrane [23]. These transporters are generally classified into three different groups; monocarboxylate transporter family (MCT8 and MCT10), organic anion transporting polypeptide family (OATP1C1) and Amino Acid Transporters [23]. The expression of these transporters are tissue specific, and represent one of the control mechanisms of THs action at cells level [24].

In the serum, two major THs are present; T4 which represents a prohormone that is the major product of thyroid gland, and T3 is the active form of the hormone at cellular level produce from thyroid gland at low level but in the tissue level especially liver and kidney T4 is converted into T3 by effects of family of enzyme called Deiodinases [25]. The effects of deiodinases enzymes represents another intracellular regulators of THs activity at cell level. Deiodinases are groups of selenocysteine-containing enzymes that have the ability of eliminating iodide from iodothyronines. As shown in Fig-2, three distinct classes of deiodinases been present; $5^{\circ}$-deiodinase converts $\mathrm{T} 4$ into active T3, 5-deiodinase converts T4 into inactive reverse rT3 and 5,5-deiodinase transforms T4 into inactive Diiodothyronine T2. These enzymes have a tissue specificity and ability to control THs activity independent of circulating T4 or T3 levels [26]. 


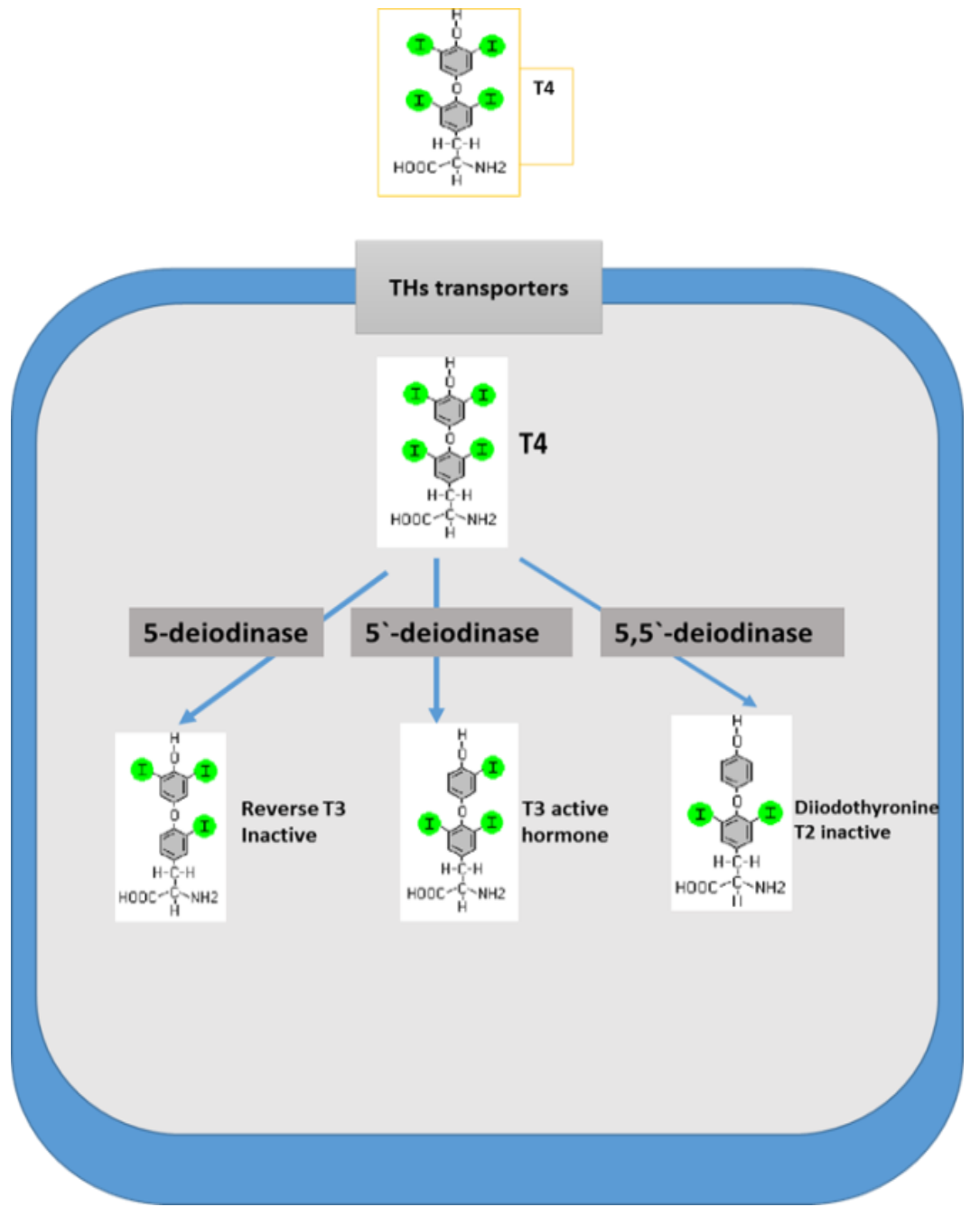

Fig-2: Schematic graph of the thyroid hormones cellular uptake and regulation by deiodinases enzymes. The entry of dominant circulatory form of THs (T4) via selective THs transporters that have tissues and cellular specificity. Within the cells, a group of deiodinases enzymes that also have tissue specificity catalyze the conversion of T4 into different form of THs with variable activity

THs intracellular receptors are nuclear receptors that act as T3 mediated gene transcription. Generally, in tissues specific expression mode two types of THs receptors been described; TR $\alpha$ and TR $\beta$. In bone and heart tissues $\mathrm{TR} \alpha$ is the predominant while in liver and kidney, the TR $\beta$ been the predominant receptors. In the nucleus, THs receptors bind to $\mathrm{T} 3$ in a specific point of DNA (THs response elements), and exert their action on gene transcription through hormone-responsive nuclear transcription factors NR1A1 and NR1A2 [27]. Additionally, THs can act directly in the cytoplasm, on mitochondria or at the plasma membrane freely without binding to the receptors affecting membrane permeability, mitochondrial activity and cytoplasmic enzymes activity [28]. Thus, THs activity could be modified independent on serum level of T4 and T4 at cellular level in different tissues by selective transport across plasma membrane, effects of tissue specific deiodinases enzymes and tissue specific binding of THs to their receptors.

\section{Effects of thyroid hormones on intestinal Glucose Absorption}

Physiologically, glucose and other monosaccharides are hydrophilic molecules, they cannot passively cross the cell membrane, and therefore their absorption require transporters. Glucose absorption from intestine lumen is mediated by apical sodium glucose cotransporter 1 (SGLT1), which carries 1 glucose molecule with two molecules of $\mathrm{Na}^{+}$ions into the epithelial cell. The concentration of $\mathrm{Na}^{+}$ions within the enterocytes kept by the effect of apical $\mathrm{Na}+\mathrm{H}+$ exchanger and $\mathrm{Na}+\mathrm{K}+$ ATPase pump of the basolateral side [29]. Then, glucose is transported via basolateral glucose transporter 2 (GLUT2) of the epithelial cells [30]. In state of high luminal sugar concentrations, GLUT2 can help in glucose absorption by translocation into apical brush border [31]. At intestinal epithelial cell level, a direct effects of THs been observed and associated with increased activity of $\mathrm{Na}^{+}$glucose cotransporter (SGLT-1). The increased activity of (SGLT-1) leads to the increased glucose absorption in 
response to thyroid hormone is due to enhanced $\mathrm{Na}^{+}-\mathrm{K}^{+}$ ATPase activity [32].

It is accepted for long time that absorption of glucose is increased in hyperthyroidism and decreased in hypothyroidism [33]. In hyperthyroidism, the increased glucose absorption occurs as a results of faster rate of stomach emptying and increased portal blood flow [9]. Furthermore, increased intestinal motility and increased smooth muscle activity was observed in state of high THs [34]. In hypothyroidism, the reverse is observed that the sluggish intestinal motility [35].

\section{Effects of thyroid hormones on liver Glucose metabolism}

It is well-documented that THs affect glucose homeostasis. In the liver, THs have an influence on carbohydrate metabolism [36]. For better Understanding of the effects of THs on liver glucose metabolism, through reviewing of the pathways of hepatic glucose metabolism would be of value. The absorbed glucose from the intestine and via portal circulation is firstly handled by liver cells. Physiologically, liver is the primary site of glucose utilization after meals. About up to $60 \%$ of the ingested glucose is dealt with within the liver post-prandially [37]. In the hepatocyte, the entered glucose is firstly phosphorylated to glucose 6-phosphate, which represents the key molecule of variable metabolic pathways such as; oxidative pathway, the pentose phosphate pathway and glycogen synthesis. Furthermore, liver is the primary site of converting excess glucose into fatty acids. Finally, liver represents the only organ that could supply the circulation with glucose during fasting either through glycogenolysis of the stored glycogen or via gluconeogenesis (synthesis of glucose from non-carbohydrate precursors such as glycerol, lactate and alanine) [38].

\section{Transport of Glucose into and from the hepatocyte}

Glucose transports into and out of the hepatocytes via glucose transporter-2 (GLUT2), which passively transport glucose into and out of liver cells in an insulin independent manner. Thus, hyperglycaemia is the only determinant of increase glucose uptake in hepatocytes [39]. Animal studies documented that GLUT2 expression in hepatocytes is regulated by THs [40-42]. GLUT2 was shown to be expressed of about double amount in hyperthyroidism compared to hypothyroid animal liver cells and treatment of hypothyroid animal with thyroxin shown to acutely enhance GLUT2 mRNA levels in animal hepatocytes [40]. Furthermore, in human hepatic glucose output which is also facilitated by GLUT2 was shown to increase in hyperthyroidism [7]. In addition, liver GLUT2 expression was shown to be reduced in adult with hypothyroidism [43]. In conclusion, glucose transport in hepatocytes is shown to be affected by THs.
Hepatic Glucose phosphorylation by glucokinase

The Entered glucose into the hepatocyte is phosphorylated by isoenzymes of hexokinase and in human liver, cells and glucokinase is the dominant isoform. Phosphorylating activity of glucokinase responsible for about $95 \%$ of the glucose phosphorylation resulting in glucose 6-phosphate and the remaining $5 \%$ is accounting for the other isoenzymes of hexokinase [44]. The net function of phosphorylation process by this enzyme is promotion of glucose uptake, maintaining hepatic glucose balance and utilization primarily through glycogen synthesis by keeping proper glucose gradient for influx into the cell [45]. Glucokinase expression is induced by insulin and suppressed by glucagon [46]. Regarding THs effects, Circulating levels of T3 induced glucokinase activity in animal liver [47]. Furthermore, THs shown to potentiate the effects of insulin on increasing glucokinase activity in animal liver in dose dependent manner but this increase shown to be abolished in hypothyroid animals [48]. Another study conducted by Sibrowski et al., [49]showed that hepatic glucokinase activity was shown to be impaired in hypothyroid animal's liver cells and the response to insulin induction of the enzyme activity was limited and these abnormalities shown to be corrected by T3 induction. In the same study comparing enzyme activity in different thyroid status showed that, glucokinase basal activity and after insulin stimulation shown to be more than 2 fold in hyperthyroid compared to hypothyroid animals [49]. In summary, THs shown to play pivotal role in glucokinase activity in hepatocytes.

\section{Hepatic Glucose 6-phosphate metabolism}

The produced Glucose 6-phosphate within the hepatocyte may go into three known metabolic pathways, namely glycogen synthesis, the pentose phosphate pathway and glycolysis [38].

\section{Glycogen Synthesis}

Glycogen synthesis is the major pathway of glucose utilization and storage within human liver. In the hepatocyte, glucose molecules being stored as a glycogen primarily from food derived glucose post prandially which account for about (73\%), and about $(27 \%)$ the glucose produced from gluconeogenesis in both fast and fed states [50].

Biochemically, glycogenesis involves three essential steps for converting Glucose 6-phosphate into mature glycogen. First, Activation of Glucose, isomerization of Glucose 6-phosphate to Glucose 1phosphate by the enzyme (phosphoglucomutase-1), then formation of UDP-glucose that acts as glucose donor for the formed glycogen. The energy derived for the formation of UDP-glucose is obtained from high energy nucleotide UTP. The second step is the elongation process. The glucose part of the UDPglucose is moved to glycogenin (glycogen primer) which accept glucose and added it to the nonreducing 
end forming 1, 4 glycosidic linkage and liberates UDP and this process is achieved by the enzyme glycogen synthase (highly regulated enzyme). The third step is a branching process, when the former glycogen residues reach 11-12 glucose a branch of 6 to 8 glucose residues are added by addition of alpha-1, 6 linkages by the branching enzyme [51].

It is well-known that glycogen synthesis is hormonally controlled. Insulin, glucagon, glucocorticoids, and catecholamines are the main hormones that metabolically controlled glycogen synthesis [52]. The effects of THs on glycogen synthesis were thoroughly studied. Bollen and Stalmans [53] in an animal study showed that glycogen synthesis was decreased in hyperthyroid and increased in hypothyroid rats' hepatocytes, and these effects were attributed to THs action on glycogen synthase enzyme. Furthermore, THs treatment was shown to inhibit insulin stimulating glycogen synthesis by decrease gene expression of Akt 2 enzyme (a direct stimulator of glycogen synthase) in hypothyroid mice [54]. Glycogen content in hepatocyte from dogs was lower in T3 treated compared to non-T3 treated animals [55]. It was also demonstrated that in thyrotoxicosis animal model, glycogen synthesis was impaired after carbohydrates rich food [56]. These effects of THs on glycogen synthesis in hyper and hypothyroidism could contribute and even worsening hepatic insulin resistance.

\section{Pentose Phosphate Pathway}

Also known

as

HEXOSE

MONOPHOSPHATE (HMP) SHUNT PATHWAY, an important hepatic pathway of glucose metabolism occurs in hepatocytes cytoplasm. The key resultant molecules from this pathway would be ribose sugars for nucleotides synthesis and reducing equivalents (NADPH) for synthesis of fatty acids [57].

Biochemically, Pentose phosphate pathway in the liver cells has two phases; oxidative and nonoxidative. The results of oxidative phase would be one molecule of pentose phosphate and 2 molecules of NADPH. Two important enzymes are regulating this phase; the firs, NADP+ dependent Glucose-6-phosphate dehydrogenase (GPD) and 6-phosphogluconate dehydrogenase. In the non-oxidative phase intermediates of glycolysis produced from the pentose phosphates [58]. The effects of THs on enzyme activity of this pathway were studied on lab animals. Both the activity the oxidative and non-oxidative enzymes of Pentose phosphate pathway shown to reduce in hypothyroid animals and the activity return to normal with thyroxin treatment [59]. The revers was observed in hyperthyroidism, there was an increased activity of the oxidative phase enzymes in hyperthyroidism animal models [60]. Furthermore, the enzymes of the pentose phosphate pathway were shown to increase in response to thyroxin treatment or feeding animals with Iodinated Casein [61]. These changes of this pathway's enzymes brought the attention about the effects of THs on different metabolic abnormalities associated with abnormal glucose metabolism.

\section{Hepatic Glucose oxidation}

Glycolysis and tricarboxylic acid (TCA) cycle followed by electron transport chain in the mitochondria represent the end results of complete glucose oxidation. Notably, liver is not predominant oxidative organ like that observed in brain and skeletal muscle that responsible for burning furthermost glucose [62].

In the cytosol of hepatocytes, Glycolysis takes place and small amount of energy results (2 ATP) with generation of NADH without the need of oxygen. A series of biochemical interaction of conversion of glucose into 2 pyruvate molecules [63].

The effects of THs on hepatocytes glycolysis been studied. An interesting study conducting by Gregory and Berry [64] on rats liver cells showed that no differences in rate of glycolysis in different thyroid status (euthyroidism, hypothyroidism and hyperthyroidism). This study measured the cytoplasmic reducing equivalents as a major products of glycolysis. However the recorded enhancements of expression and activity of the enzymes of glycolytic pathway by THs $[65,66]$ but no differences in glycolysis rate been observed. This greatly support the assumption that the major effects of THs been on mitochondrial oxidation of glucose.

The next stage of glucose oxidation is complete conversion of pyruvate to acetyl-CoA in the mitochondria utilized by Pyruvate dehydrogenase (PDH) enzyme. This enzyme complex plays an invaluable role in hepatic glucose metabolism and represents the major regulatory control point for both substrate and hormones [67]. Activation of this enzyme complex leading to two major effects; first, promotion of glucose oxidation, and secondly, redirection of conversion of pyruvate to malonyl-CoA (fatty acid synthesis). Inactivation of Pyruvate dehydrogenase is leading to decrease glucose oxidation and enhancement of gluconeogenesis by conversion of available pyruvate to glucose [68]. Biochemically, Pyruvate dehydrogenase is activated by $\mathrm{Ca}^{++}, \mathrm{Mg}^{++}$and AMP (decreased energy availability), while it is inactivated by phosphorylation of the $\alpha$-subunit of the enzyme complex by pyruvate dehydrogenase kinase enzyme. This kinase responsible for keeping the enzyme in an inactive form (phosphorylated) under conditions of increase energy availability represented by abundance of acetyl-CoA and $\mathrm{NADH}$ that results from glycolysis and fatty acids oxidations [69].

In hypothyroid rats liver cells, the pyruvate dehydrogenase enzyme expression and activity seems to be significantly reduced compared to euthyroid 
animals, while no differences in enzyme expression and activity were observed between hyper and euthyroid animals liver cells [70]. Furthermore, Hyperthyroidism is shown to enhanced rate of fatty acid oxidation in liver cells that leads to inactivation of the pyruvate dehydrogenase enzyme complex due to abundance of acetyl-CoA and NADH [71]. In addition study conducted by Priestman et al., [72] showed that, the long-term effect of hyperthyroidism on rat's hepatocytes is significant decrease in activity of pyruvate dehydrogenase enzyme which was accounted to increase level and activity of pyruvate dehydrogenase kinase enzyme. The decrease level and activity of the pyruvate dehydrogenase enzyme that observed in hyperthyroidism would promptly lead to enhancement of conversion of pyruvate to glucose by activation of gluconeogenesis which could contribute to increase level of glycaemia.

\section{Hepatic mitochondrial oxidation TCA cycle}

Inside the mitochondria, the generated acetylCoA is condensed with oxaloacetate to result in citrate which represents the control point molecule. In state of energy demand, citrate completes the whole circle of the TCA cycle generating reducing equivalents and ATP. When the energy state of the cell is in excess the citrate exits the mitochondrion into cytoplasm [73].

Mitochondria plays a central role in energy generation and completion of glucose oxidation within the hepatocytes. THs modulates mitochondrial activity in direct or in direct ways. The direct way been through specific binding site inside the mitochondria, while the indirect represent the effects of THs on any other site within the cell such as nucleus [74]. THs represents the major molecules that control mitochondrial biogenesis in mammals. Early Studies showed that in hypothyroidism, both the number and size of mitochondria are decreased [75]. Furthermore, THs increase expression and production of mitochondrial proteins [76]. Conversely, in hyperthyroidism metabolic process both catabolic and anabolic are increased and energy generated from carbohydrate metabolism are accelerated [77]. Both the numbers and activity of mitochondria were shown to highly increase in a state of high THs [78].

THs induces expressions of several genes responsible for mitochondrial activity. For example, PGC-1alpha (peroxisome proliferator-activated receptor gamma coactivator 1alpha) expression and activity shown to be induced by THs in hepatocytes [79]. The role of PGC-1alpha has a profound effects on mitochondrial biogenesis [74]. Thus, THs play a pivotal role in energy production from glucose oxidation.

\section{Glucose production by the liver}

The human liver represents the major organ that has the ability to produce glucose and to release it to the blood stream to be used by other tissues. Two important processes were performed by the liver; the first is by glycogenolysis (breaking down of glycogen to glucose especially after short-term periods of fasting). The second; is by gluconeogenesis (de novo synthesis of glucose usually after prolonged periods of fasting when the hepatic glycogen store depleted) [80]. These processes are highly controlled by hormones. Insulin promotes glycogen synthesis and inhibits glycogenolysis and reduces gluconeogenesis that collectively reduce hepatic glucose production. On the other hand, glucagon is transiently induced glycogenolysis and reduce glycogen content that leading to raised glucose production [81].

\section{Gluconeogenesis}

A process of production of glucose molecules from non-carbohydrate precursors) such as glycerol, lactate and alanine) within the cells represents the gluconeogenesis pathway. It takes place in both mitochondria and cytoplasm. The key enzymes of gluconeogenesis are Pyruvate carboxylase, Phosphorene pyruvate carboxy kinase (PEPCK), Fructose-1-6-bisphosphatase and Glucose-6phosphatase [82]. The first two enzymes served the conversion of pyruvate in mitochondria to phosphoenol pyruvate in the cytoplasm. The formed phosphoenol pyruvate go through further reactions with the catalysis action of glycolytic enzymes to produce fructose-1, 6bisphosphate. Then fructose-6-phosphate is resulted from dephosphorylation reaction followed by isomerization to glucose-6-phosphate. Lastly free glucose is produced by dephosphorylation with the effect of last enzyme of the reaction Glucose-6phosphatase [51]. The gluconeogenesis pathway is highly regulated by hormones. Glucagon and glucocorticoid have stimulatory effects while insulin acting as a major inhibitory hormone.

The role of THs in Gluconeogenesis is noticeable. Thyroid stimulating hormone TSH directly affects hepatic Gluconeogenesis pathway through promoting the expressions of genes involved in this pathway such as; PEPCK and G6Pase genes that subsequently induced hepatic gluconeogenesis [83]. In addition, TSH shown to be associated with insulin resistance [84]. THs directly affects hepatic gluconeogenesis.

Liver Pyruvate Carboxylase and Phosphoenolpyruvate Carboxykinase Activities were shown to be impaired in hypothyroid animal (30\% of the enzyme activity in euthyroid state), that shown to be corrected by T3 treatment [85]. Furthermore, glucagonstimulation of gluconeogenesis shown to be reduced in hypothyroid animal liver cells [85].

\section{Pyruvate} carboxylase and phosphoenolpyruvate carboxykinase (PEPCK) activities, the key enzymes of gluconeogenesis, were shown to highly increased in hyperthyroidism, and the 
extra-thyroid hormones in hyperthyroidism shown to have permissive effect of glucagon-stimulation of gluconeogenesis [86].

The direct effects of THs are by enhancement of the promotion of expression of the genes of gluconeogenesis (PEPCK and G6Pase genes) and by modulating a promoting factors that act as transcriptional factors of gluconeogenesis [87]. In hyperthyroidism, due to increase metabolic rate and resting energy expenditure that associated with increased demand and then promotes gluconeogenesis [88]. The reverse was observed in hypothyroidism.

\section{Glycogenolysis}

Glucose is produced from hepatic glycogen after period of fasting, and this glucose can be released to the circulation to be used by other tissues. Glycogenolysis is a cytoplasmic pathway, under control of two main enzymes; Glycogen phosphorylase and glycogen debranching enzyme [38]. The effects of THs on Glycogenolysis in hepatocytes could be accounted due to different mechanisms. The increased energy expenditure by THs leading to drop in ATP and an elevation of ADP, AMP concentrations within the hepatocytes that might act on enzymes involved in glycogenolysis [89]. Furthermore, THs activated receptor shown to potentiate the effects of adrenergic agonists and glucagon hormones and thereby phosphorylation and activation of the glycogen phosphorylase [90]. Moreover, all the processes that shown to increase metabolic energy production and their enzymes were shown to be induced by THs [91, 92]. Thus, in hypothyroidism glycogenolysis seems to reduced compared to euthyroid [93, 94]. In contrary, in hyperthyroidism the rate and the enzymes of glycogenolysis seems to be induced [95-97].

\section{CONCLUSIONS}

Thyroid hormones represents a major regulator of metabolism especially glucose metabolism. These hormones achieve their effects through promotion or demotion of expression or activation of enzymes responsible for regulation of different metabolic pathways. Generally, excess thyroid hormones in hepatocytes been associated with increased glucose uptake, consumption and production. The reverse was generally accepted for low thyroid hormone level that shown to decrease glucose production and utilization. These changes during thyroid disorders is associated with abnormal metabolism that could disturb the fine balance for metabolic pathways and contributes to cause or exacerbate other concomitant disease such as diabetes and metabolic syndrome.

Disclaimer: The work presented in this review is our own work and not been submitted previously to any other place.
Conflicts of interest: We have no potential conflicts of interest relevant to this article.

Source of Support: This work is totally funded by the participated authors.

\section{ACKNOWLEDGMENTS}

We would like to show deep thanks to the teaching staff of the Department of Chemistry and Biochemistry, College of Medicine, Mustansiriyah University for their scientific supports.

\section{REFERENCES}

1. Mullur, R., Liu, Y. Y., \& Brent, G. A. (2014). Thyroid hormone regulation of metabolism. Physiological reviews. 94:355-82.

2. Teixeira PdFdS, Dos Santos, P. B., \& Pazos-Moura, C. C. (2020). The role of thyroid hormone in metabolism and metabolic syndrome. Therapeutic Advances in Endocrinology and Metabolism. 11:2042018820917869.

3. Kim, M. J., Cho, S. W., Choi, S., Ju, D. L., Park, D. J., \& Park, Y. J. (2018). Changes in body compositions and basal metabolic rates during treatment of Graves' disease. International journal of endocrinology. 2018.

4. Chng, C. L., Lim AYY., Tan HC., Kovalik J. P., \& Tham, K. W. (2016). Physiological and metabolic changes during the transition from hyperthyroidism to euthyroidism in Graves' disease. Thyroid. 26:1422-30.

5. Brent, G. A., \& Weetman, A. P. (2016). Hypothyroidism and thyroiditis. In Williams textbook of endocrinology:416-48: Elsevier. Number of 416-48 pp.

6. McAninch, E. A., \& Bianco, A. C. (2014). Thyroid hormone signaling in energy homeostasis and energy metabolism. Annals of the New York Academy of Sciences. 1311:77.

7. Jang, J., Kim, Y., Shin, J., Lee, S. A., Choi, Y., \& Park, E. C. (2018). Association between thyroid hormones and the components of metabolic syndrome. BMC endocrine disorders. 18:1-9.

8. Albayati, A. A., \& Hussein, A. A. R. (2020). Role of fetuin-A and hsCRP in CVD risk in hypothyroidism Iraqi women. Biochemical and Cellular Archives. 20:3877-81.

9. Tarnowska, A., Walicka, M., \& Franek, E. (2017). Disorders of carbohydrate metabolism in endocrinopathies. Postępy Nauk Medycznych.

10. Brent, G. A. (2012). Mechanisms of thyroid hormone action. The Journal of clinical investigation 122:3035-43.

11. Duntas, L. H., Orgiazzi, J., \& Brabant, G. (2011). The interface between thyroid and diabetes mellitus. Clinical endocrinology, 75:1-9.

12. Potenza, M., Via, M. A., Yanagisawa, R. T. (2009). Excess thyroid hormone and carbohydrate metabolism. Endocrine Practice. 15:254-62.

13. 13. Dimitriadis G, Raptis S. 2001. Thyroid hormone excess and glucose intolerance. Experimental and Clinical Endocrinology \& Diabetes 109:S225-S39 
14. Maratou, E., Hadjidakis, D. J., Peppa, M., Alevizaki, M., \& Tsegka, K. (2010). Studies of insulin resistance in patients with clinical and subclinical hyperthyroidism. European journal of endocrinology. 163:625.

15. Havekes, B., \& Sauerwein, H. P. (2010). Adipocytemyocyte crosstalk in skeletal muscle insulin resistance; is there a role for thyroid hormone? Current Opinion in Clinical Nutrition \& Metabolic Care. 13:641-6.

16. Visser, T. J. (2018). Regulation of thyroid function, synthesis and function of thyroid hormones. Thyroid Diseases: Pathogenesis, Diagnosis and Treatment; Vitti, P., Hegedus, L., Eds:1-30.

17. Carvalho D. P., \& Dupuy, C. (2017). Thyroid hormone biosynthesis and release. Molecular and cellular endocrinology 458:6-15

18. Drigo, R. A., Fonseca, T. L., Werneck-de-Castro J. P. S., \& Bianco, A. C. (2013). Role of the type 2 iodothyronine deiodinase (D2) in the control of thyroid hormone signaling. Biochimica et Biophysica Acta (BBA)-General Subjects. 1830:3956-64.

19. Hoffenberg, R., \& Ramsden, D. (1983). The transport of thyroid hormones. Clinical Science. 65:337-42.

20. Koibuchi, N. (2018). Molecular Mechanisms of Thyroid Hormone Synthesis and Secretion. Principles of Endocrinology and Hormone Action, 73-81.

21. Ortiga- Carvalho, T. M., Chiamolera, M. I., PazosMoura, C. C., \& Wondisford, F. E. (2011). Hypothalamus- pituitary- thyroid axis. Comprehensive Physiology. 6:1387-428.

22. Gereben, B., Zeöld, A., Dentice, M., Salvatore, D., \& Bianco, A. (2008). Activation and inactivation of thyroid hormone by deiodinases: local action with general consequences. Cellular and Molecular Life Sciences, 65:570-90.

23. Groeneweg, S., van Geest, F. S., Peeters, R. P., Heuer, H., \& Visser, W. E. (2020). Thyroid hormone transporters. Endocrine reviews, 41:146-201.

24. Braun, D., \& Schweizer, U. (2018). Thyroid hormone transport and transporters. Vitamins and hormones, 106:19-44.

25. Russo, S. C., Salas-Lucia, F., \& Bianco, A. C. (2021). Deiodinases and the Metabolic Code for Thyroid Hormone Action. Endocrinology.

26. Luongo, C., Dentice, M., \& Salvatore, D. (2019). Deiodinases and their intricate role in thyroid hormone homeostasis. Nature Reviews Endocrinology, 15:479-88.

27. Yen, P. M., Ando, S., Feng, X., Liu, Y., Maruvada, P., \& Xia, X. (2006). Thyroid hormone action at the cellular, genomic and target gene levels. Molecular and cellular endocrinology, 246:121-127.

28. Davis, P. J., Goglia, F., \& Leonard, J. L. (2016). Nongenomic actions of thyroid hormone. Nature Reviews Endocrinology, 12:111-121.

29. Augustin, R., \& Mayoux, E. (2014). Mammalian sugar transporters. In Glucose homeostasis: IntechOpen. Number of.
30. Wright, E. M., Loo, D. D., \& Hirayama, B. A. (2011). Biology of human sodium glucose transporters. Physiological reviews, 91:733-94.

31. Zheng, Y., Scow, J. S., Duenes, J. A., \& Sarr, M. G. (2012). Mechanisms of glucose uptake in intestinal cell lines: role of GLUT2. Surgery, 151:13-25.

32. Matosin-Matekalo, M., Mesonero, J. E., Delezay, O., Poiree, J. C., Ilundain, A. A., \& Brot-Laroche, E. (1998). Thyroid hormone regulation of the $\mathrm{Na}+$ /glucose cotransporter SGLT1 in Caco-2 cells. Biochemical Journal 334:633-640.

33. Gierach, M., Gierach, J., \& Junik, R. (2014). Insulin resistance and thyroid disorders. Endokrynologia Polska, 65:70-76.

34. Brown, R. B. (1940). The Gastro-intestinal Tract in Hyperthyroidism. Graduate School of Arts and Sciences, University of Pennsylvania.

35. Middleton, W. (1971). Thyroid hormones and the gut. Gut, 12:172.

36. Crunkhorn, S., \& Patti, M. E. (2008). Links between thyroid hormone action, oxidative metabolism, and diabetes risk? Thyroid. 18:227-37.

37. Ferrannini, E., Bjorkman, O., Reichard, G. A., Pilo, A., Olsson, M., Wahren, J., \& DeFronzo, R. A. (1985). The disposal of an oral glucose load in healthy subjects: a quantitative study. Diabetes, 34(6), 580-588.

38. Adeva-Andany MM, Pérez-Felpete N, FernándezFernández C, Donapetry-García C, Pazos-García C. 2016. Liver glucose metabolism in humans. Bioscience reports 36

39. Adkins, A., Basu, R., Persson, M., Dicke, B., Shah, P., Vella, A., ... \& Rizza, R. (2003). Higher insulin concentrations are required to suppress gluconeogenesis than glycogenolysis in nondiabetic humans. Diabetes, 52(9), 2213-2220.

40. Weinstein SP, O'Boyle E, Fisher M, Haber RS. 1994. Regulation of GLUT2 glucose transporter expression in liver by thyroid hormone: evidence for hormonal regulation of the hepatic glucose transport system. Endocrinology, 135:649-54.

41. Kemp, H. F., Hundal, H. S., \& Taylor, P. M. (1997). Glucose transport correlates with GLUT2 abundance in rat liver during altered thyroid status. Molecular and cellular endocrinology, 128:97-102.

42. Mokuno, T., Uchimura, K., Hayashi, R., Hayakawa, N., Makino, M., Nagata, M., ... \& Itoh, M. (1999). Glucose transporter 2 concentrations in hyper-and hypothyroid rat livers. Journal of Endocrinology, 160(2), 285-289.

43. Godini A, Ghasemi A, Zahediasl S. 2015. The possible mechanisms of the impaired insulin secretion in hypothyroid rats. PloS one, 10:e0131198

44. Iynedjian PB, Marie S, Gjinovci A, Genin B, Deng S-P, et al. 1995. Glucokinase and cytosolic phosphoenolpyruvate carboxykinase (GTP) in the human liver. Regulation of gene expression in cultured hepatocytes. The Journal of clinical investigation, 95:1966-73

45. Stoffel M, Froguel P, Takeda J, Zouali H, Vionnet N, et al. 1992. Human glucokinase gene: isolation, characterization, and identification of two missense mutations linked to early-onset non-insulin- 
dependent (type 2) diabetes mellitus. Proceedings of the National Academy of Sciences, 89:7698-702

46. Iynedjian P, Gjinovci A, Renold A. 1988 Stimulation by insulin of glucokinase gene transcription in liver of diabetic rats. Journal of Biological Chemistry, 263:740-4

47. Decaux J-F, Juanes M, Bossard P, Girard J. 1997. Effects of triiodothyronine and retinoic acid on glucokinase gene expression in neonatal rat hepatocytes. Molecular and cellular endocrinology, 130:61-7

48. Şahin, D., Altan, N., Sepici-Dinçel, A., \& Engin, A. (2019). The Effects of Thyroid Hormones on Glucokinase Enzyme Activity in diabetic Rat Liver Tissues. Tıp Fakültesi Klinikleri Dergisi, 2:105-12.

49. Sibrowski W, Müller M, Seitz H. 1981. Effect of different thyroid states on rat liver glucokinase synthesis and degradation in vivo. Journal of Biological Chemistry, 256:9490-4.

50. Woerle HJ, Meyer C, Dostou JM, Gosmanov NR, Islam N, et al. 2003. Pathways for glucose disposal after meal ingestion in humans. American Journal of Physiology-Endocrinology and Metabolism, 284:E716-E25

51. Vasudevan DM, Sreekumari S, Vaidyanathan K. 2016. Textbook of biochemistry for medical students. JP Medical Ltd.

52. Ahsan A, Khan A, Farooq MA, Naveed M, Baig MMFA, Tian W-x. 2020. Physiology of Endocrine System and Related Metabolic Disorders. In Endocrine Disrupting Chemicals-induced Metabolic Disorders and Treatment Strategies:3-41: Springer. Number of 3-41 pp.

53. Bollen M, Stalmans W. 1988. The effect of the thyroid status on the activation of glycogen synthase in liver cells. Endocrinology, 122:2915-9.

54. Feng X, Jiang Y, Meltzer P, Yen PM. 2000. Thyroid hormone regulation of hepatic genes in vivo detected by complementary DNA microarray. Molecular Endocrinology, 14:947-55.

55. Brzezińska Z, Kaciuba-Uścilko H. 1979. Low muscle and liver glycogen contents in dogs treated with thyroid hormones. Hormone and Metabolic Research, 11:675-8

56. Holness, M. J., \& Sugden, M. C. (1987). Continued glucose output after re-feeding contributes to glucose intolerance in hyperthyroidism. Biochemical journal, 247:801.

57. Liu, J., Jin, X., Zhou, F., Chen, H., Wang, W., Liu, Y., ... \& Zhang, J. (2020). Disrupted hepatic pentose phosphate pathway directly participates in and indirectly promotes CYP3A reduction: A new strategy for CYP3A- mediated drug hepatotoxicity. British journal of pharmacology, 177(7), 1538-1555.

58. Ge T, Yang J, Zhou S, Wang Y, Li Y, Tong X. 2020 The role of the pentose phosphate pathway in diabetes and cancer. Frontiers in Endocrinology, 11:365.

59. Novello F, Gumaa J, Mclean P. (1969). The pentose phosphate pathway of glucose metabolism. Hormonal and dietary control of the oxidative and non-oxidative reactions of the cycle in liver. Biochemical Journal, 111:713-25.

60. Jung, S. Y., Lee, J., \& Lee, D. H. (2020). Persistent goiter with congenital hypothyroidism due to mutation in DUOXA2 gene. Annals of Pediatric Endocrinology \& Metabolism, 25:57.

61. Freedland, R. (1965). Effects of thyroid hormones on metabolism. Effect of thyroxine and iodinated casein on liver enzyme activity. Endocrinology, 77:19-27.

62. Naifeh J, Dimri M, Varacallo M. (2017) Biochemistry, aerobic glycolysis.

63. Alemanno F. 2020. Anaerobic Glycolysis or Embden-Meyerhof Pathway. In Biochemistry for Anesthesiologists and Intensivists:7-14: Springer. Number of 7-14 pp.

64. Gregory, R. B., \& Berry, M. N. (1995). The influence of thyroid state on hepatic glycolysis. European journal of biochemistry. 229:344-8.

65. Baquer, N. Z., Cascales, M., Mclean, P., \& Greenbaum, A. L. (1976). Effects of thyroid hormone deficiency on the distribution of hepatic metabolites and control of pathways of carbohydrate metabolism in liver and adipose tissue of the rat. European journal of biochemistry, 68:403-13.

66. Höppner, W., \& Seitz, H. J. (1989). Effect of thyroid hormones on glucokinase gene transcription in rat liver. Journal of Biological Chemistry, 264:20643-7.

67. Sugden, M. C., \& Holness, M. J. (1994). Interactive regulation of the pyruvate dehydrogenase complex and the carnitine palmitoyltransferase system. The FASEB journal. 8:54-61.

68. 68. Holness MJ, Bulmer K, Smith ND, Sugden MC 2003. Investigation of potential mechanisms regulating protein expression of hepatic pyruvate dehydrogenase kinase isoforms 2 and 4 by fatty acids and thyroid hormone. Biochemical Journal 369:68795

69. Jakkamsetti, V., Marin-Valencia, I., Ma, Q., \& Pascual, J. M. (2020). Pyruvate dehydrogenase, pyruvate carboxylase, Krebs cycle, and mitochondrial transport disorders. In Rosenberg's Molecular and Genetic Basis of Neurological and Psychiatric Disease:427-36: Elsevier. Number of 427-36 pp.

70. Weinberg, M. B., \& Utter, M. F. (1979). Effect of thyroid hormone on the turnover of rat liver pyruvate carboxylase and pyruvate dehydrogenase. Journal of Biological Chemistry. 254:9492-9.

71. Holness, M. J., French, T. J., Schofield, P. S., \& Sugden, M. C. (1987). The relationship between fat synthesis and oxidation in the liver after re-feeding and its regulation by thyroid hormone. Biochemical journal. 247:621-6.

72. Priestman, D. A., Donald, E., Holness, M. J., \& Sugden, M. C. (1997). Different mechanisms underlie the long- term regulation of pyruvate dehydrogenase kinase (PDHK) by tri- iodothyronine in heart and liver. FEBS letters, 419:55-7.

73. Brownsey, R., Boone, A., Elliott, J., Kulpa, J., \& Lee, W. (2006). Regulation of acetyl-CoA carboxylase. Biochemical Society Transactions, 34:223-7.

74. Cioffi, F., Senese, R., Lanni, A., \& Goglia, F. (2013). Thyroid hormones and mitochondria: with a brief 
look at derivatives and analogues. Molecular and cellular endocrinology, 379:51-61.

75. Gustafsson, R., Tata, J., Lindberg, O., \& Ernster, L. (1965). The relationship between the structure and activity of rat skeletal muscle mitochondria after thyroidectomy and thyroid hormone treatment. The Journal of cell biology. 26:555-78.

76. Mutvei, A., Kuzela, S., \& Nelson, B. D. (1989). Control of mitochondrial transcription by thyroid hormone. European journal of biochemistry, 180:235-40.

77. Sestoft, L. (1980). Metabolic aspects of the calorigenic effect of thyroid hormone in mammals. Clinical endocrinology. 13:489-506.

78. Soboll, S. (1993). Thyroid hormone action on mitochondrial energy transfer. Biochimica et Biophysica Acta (BBA)-Bioenergetics. 1144:1-16.

79. Thakran, S., Sharma, P., Attia, R. R., Hori, R. T., Deng, X., Elam, M. B., \& Park, E. A. (2013). Role of sirtuin 1 in the regulation of hepatic gene expression by thyroid hormone. Journal of Biological Chemistry, 288(2), 807-818.

80. Petersen, M. C., Vatner, D. F., \& Shulman, G. I. (2017). Regulation of hepatic glucose metabolism in health and disease. Nature reviews endocrinology 13:572-87.

81. Santoleri, D., \& Titchenell, P. M. (2019). Resolving the paradox of hepatic insulin resistance. Cellular and molecular gastroenterology and hepatology 7:447-56.

82. Miyamoto, T., \& Amrein, H. (2017). Gluconeogenesis: An ancient biochemical pathway with a new twist. Fly, 11:218-23.

83. Li, Y., Wang, L., Zhou, L., Song, Y., Ma, S., Yu, C., ... \& Gao, L. (2017). Thyroid stimulating hormone increases hepatic gluconeogenesis via CRTC2. Molecular and cellular endocrinology, 446, 70-80.

84. 84. Galofré J, Pujante P, Abreu C, Santos S, GuillenGrima F, et al. 2008. Relationship between thyroidstimulating hormone and insulin in euthyroid obese men. Annals of Nutrition and Metabolism 53:188-94

85. Menahan, L. A., \& Wieland, O. (1969). The role of thyroid function in the metabolism of perfused rat liver with particular reference to gluconeogenesis. European journal of biochemistry, 10(1), 188-194.

86. Shrago, E., Lardy, H. A., Nordlie, R. C., \& Foster, D. O. (1963). Metabolic and hormonal control of phosphoenolpyruvate carboxykinase and malic enzyme in rat liver. Journal of Biological Chemistry, 238(10), 3188-3192.

87. Suh, J. H., Sieglaff, D. H., Zhang, A., Xia, X., Cvoro, A., Winnier, G. E., \& Webb, P. (2013). SIRT1 is a direct coactivator of thyroid hormone receptor $\beta 1$ with gene-specific actions. PloS one, 8(7), e70097.

88. Brent, G. A. (2008). Graves' disease. New England Journal of Medicine. 358:2594-605.

89. Nebioglu, S., Wathanaronchai, P., Nebioglu, D., Pruden, E. L., \& Gibson, D. M. (1990). Mechanisms underlying enhanced glycogenolysis in livers of 3, 5, 3'-triiodothyronine-treated rats. American Journal of Physiology-Endocrinology and Metabolism. 258:E109-E16.

90. Exton, J. H., Cherrington, A. D., Blackmore, P. F., Dehaye, J. P., Strickland, W. G., Jordan, J. E., \& Chrisman, T. D. (1981). Hormonal regulation of liver glycogen metabolism. Cold Spring Harbor Conference on Cell Proliferation.

91. Battarbee, H. D. (1974). The effects of thyroid state on rat liver glucose-6-phosphatase activity and glycogen content. Proceedings of the Society for Experimental Biology and Medicine, 147(2), 337343.

92. Nordlie, R. C. (1974). Metabolic regulation by multifunctional glucose-6-phosphatase. Current topics in cellular regulation, 8:33-117.

93. Storm, H., Van Hardeveld, C., \& Kassenaar, A. A. (1984). The influence of hypothyroidism on the adrenergic stimulation of glycogenolysis in perfused rat liver. Biochimica et Biophysica Acta (BBA)General Subjects, 798(3), 350-360.

94. Ali, M., Cantau, B., \& Clos, J. (1989). Glycogenolytic responsiveness to glucagon, epinephrine, vasopressin and angiotensin II in the liver of developing hypothyroid rats. A comparative study of in vitro hormonal binding and in vivo biological response. Journal of developmental physiology, 11:360-7.

95. Mirsky, I. A., \& Broh-Kahn, R. (1936). The effect of experimental hyperthyroidism on carbohydrate metabolism. American Journal of Physiology-Legacy Content. 117:6-12.

96. Brenta, G. (2010). Diabetes and thyroid disorders. The British Journal of Diabetes \& Vascular Disease, 10:172-7.

97. Kapadia, K. B., Bhatt, P. A., \& Shah, J. S. (2012). Association between altered thyroid state and insulin resistance. Journal of pharmacology \& pharmacotherapeutics, 3:156. 